\title{
The Law of Iterated Logarithm for Autoregressive Processes
}

\author{
Yan Wang, ${ }^{1}$ Mingzhi Mao, ${ }^{2}$ Xiaohua $\mathrm{Hu}^{3,4}$ and Tingting $\mathrm{He}^{3}$ \\ ${ }^{1}$ National Engineering Research Center for E-Learning, Central China Normal University, Wuhan 430079, China \\ ${ }^{2}$ School of Mathematics and Physics, China University of Geosciences, Wuhan 430074, China \\ ${ }^{3}$ Department of Computer Science, Central China Normal University, Wuhan 430079, China \\ ${ }^{4}$ College of Computing and Informatics, Drexel University, Philadelphia, PA, USA
}

Correspondence should be addressed to Yan Wang; shuxuewy@163.com

Received 24 July 2014; Accepted 24 August 2014; Published 5 November 2014

Academic Editor: Quanxin Zhu

Copyright (C) 2014 Yan Wang et al. This is an open access article distributed under the Creative Commons Attribution License, which permits unrestricted use, distribution, and reproduction in any medium, provided the original work is properly cited.

This paper mainly discusses some dynamics asymptotic properties of autoregressive processes. By using the $m$-dependence of random variables, we prove the least squares (LS) estimator of the unknown parameters satisfies the law of iterated logarithm.

\section{Introduction}

Autoregressive process is a basic model in engineering, insurance, and business. It is a representation of a type of random process; as such, it describes certain time-varying processes in nature, engineering, and so forth. The autoregressive model specifies that the output variable depends linearly on its own previous values. It is a special case of the more general autoregressive-moving average (ARMA) model of time series. For example, in engineering one considers a dam, with input of random amounts at random times, and a steady withdrawal of water for irrigation or power usage. This model has a Markovian representation. It is well known that stability problem is an important interest topic, which explains why the stochastic systems always stay in "reasonable values": the dam does not overflow. The parameters in the process have a direct relation to the system stability, so the estimation of fixed coefficients and its dynamics behaviors are very useful in engineering. There was a rich literature which focused on the research (see Mann and Wald [1], Anderson [2], Menneteau [3], Hwang and Choi [4], Hwang and Baek [5], and Miao and Shen [6]). Recently, some attention has been directed to random coefficient autoregressive models. This way of handling the data allows for large shocks in the dynamic structure of the model and also for some flexibility in the features of the volatility of the series, which are not available in fixed coefficient autoregressive models.
For simplicity, in this paper, we are concerned with some dynamics behaviors (in probability language, we call it the law of iterated logarithm) of the parameters estimation for the linear autoregressive model which is of practical importance, by a different technique from the standard method. Define the following first order autoregressive process $(\mathrm{AR}(1))\left\{X_{t}, t \geq\right.$ $1\}$ by

$$
X_{t}=\beta_{0}+\beta_{1} X_{t-1}+\epsilon_{t}, \quad\left|\beta_{1}\right|<1,
$$

where the initial value $X_{0}$ is not necessarily zero and $\left\{\epsilon_{t}\right\}$ is a sequence of independent and identically distributed (i.i.d.) random errors with mean zero and variance $\sigma^{2}$. Based on the observations $X_{1}, X_{2}, \ldots, X_{n}$, the least squares (LS) estimators $\left(\widehat{\beta}_{0}^{(n)}, \widehat{\beta}_{1}^{(n)}\right)^{T}$ of $\left(\beta_{0}, \beta_{1}\right)^{T}$ are given by

$$
\begin{aligned}
& \left(\begin{array}{l}
\widehat{\beta}_{0}^{(n)} \\
\widehat{\beta}_{1}^{(n)}
\end{array}\right) \\
& =\left(\begin{array}{cc}
n & \sum_{t=1}^{n} X_{t-1} \\
\sum_{t=1}^{n} X_{t-1} & \sum_{t=1}^{n} X_{t-1}^{2}
\end{array}\right)^{-1}\left(\begin{array}{c}
\sum_{t=1}^{n} X_{t} \\
\sum_{t=1}^{n} X_{t} X_{t-1}
\end{array}\right) .
\end{aligned}
$$


By a simple calculation, we have

$$
\begin{aligned}
& \widehat{\beta}_{0}^{(n)}-\beta_{0} \\
& =\frac{\left(\sum_{t=1}^{n} X_{t-1}^{2}\right)\left(\sum_{t=1}^{n} \epsilon_{t}\right)-\left(\sum_{t=1}^{n} X_{t-1}\right)\left(\sum_{t=1}^{n} \epsilon_{t} X_{t-1}\right)}{n \sum_{t=1}^{n} X_{t-1}^{2}-\left(\sum_{t=1}^{n} X_{t-1}\right)^{2}} \\
& \widehat{\beta}_{1}^{(n)}-\beta_{1} \\
& =\frac{-\left(\sum_{t=1}^{n} X_{t-1}\right)\left(\sum_{t=1}^{n} \epsilon_{t}\right)+n \sum_{t=1}^{n} \epsilon_{t} X_{t-1}}{n \sum_{t=1}^{n} X_{t-1}^{2}-\left(\sum_{t=1}^{n} X_{t-1}\right)^{2}}
\end{aligned}
$$

It is well known that, in the stable (or, in other words, asymptotically stationary) case, when $\left|\beta_{1}\right|<1, \widehat{\beta}_{1}^{(n)}$ is asymptotically normal under the assumption that $\left\{\epsilon_{i}\right\}$ is a sequence of i.i.d. random variables with mean zero and variance 1; namely,

$$
\sqrt{n}\left(\widehat{\beta}_{1}^{(n)}-\beta_{1}\right) \Longrightarrow \mathcal{N}\left(0,1-\beta_{1}\right)
$$

where " $\Rightarrow$ " denotes the convergence in distribution, although it does not hold uniformly for $\left|\beta_{1}\right|<1$ (see Anderson [2] and Meyn and Tweedie [7]). In the unstable (or, in other words, unit root) case when $\beta_{1}=1$, for the sequence $\widehat{\beta}_{1}^{(n)}$, we have

$$
n\left(\widehat{\beta}_{1}^{(n)}-\beta_{1}\right) \Longrightarrow \frac{\int_{0}^{1} w(t) d w(t)}{\int_{0}^{1} w(t)^{2} d t}
$$

where $\{w(t): t \in[0,1]\}$ denotes a standard Wiener process. In the explosive case, when $\left|\beta_{1}\right|>1$, the sequence $\widehat{\beta}_{1}^{(n)}$ is again not asymptotically normal. If, for example, $\epsilon_{1} \sim \mathcal{N}(0,1)$, then

$$
\beta_{1}^{n}\left(\widehat{\beta}_{1}^{(n)}-\beta_{1}\right) \Longrightarrow \text { Cauchy }\left(0, \beta_{1}^{2}-1\right)
$$

In general, the limit distribution depends on the distribution of $\epsilon_{1}$ (see Basawa and Brockwell [8]).

In this paper, we consider the convergence rates of $\beta_{0}^{(n)} \rightarrow$ $\beta_{0}$ and $\beta_{1}^{(n)} \rightarrow \beta_{1}$ under some conditions. By constructing an $m$-dependent random variables sequence and applying an approximation method along with a central limit theorem for random variables, we prove the LS estimators of $\beta_{0}$ and $\beta_{1}$ satisfy the law of iterated logarithm. Our results can be considered as an embodiment of Miao and Shen [6].

In the following statement, we have an assumption as follows:

(C.1) there exists a constant $\gamma>0$ such that $\mathbf{E} \exp \left\{\gamma\left|\epsilon_{t} \epsilon_{s}\right|\right\}<$ $+\infty$ for any $t \geq 1$ and $s \geq 1$.

\section{Main Results}

The main result is as follows.
Theorem 1. Assume that $\left|\beta_{1}\right|<1$. Then the law of iterated logarithm for $\left(\widehat{\beta}_{0}^{(n)}-\beta_{0}, \widehat{\beta}_{1}^{(n)}-\beta_{1}\right)^{T}$ holds; namely,

$$
\begin{gathered}
\limsup _{n \rightarrow \infty} \sqrt{\frac{n}{2 \log \log n}\left(\widehat{\beta}_{0}^{(n)}-\beta_{0}\right)} \\
=\sqrt{\sigma^{2}+\frac{\beta_{0}^{2}\left(1-\beta_{1}^{2}\right)}{\left(1-\beta_{1}\right)^{2}}}, \quad \text { a.s., } \\
\limsup _{n \rightarrow \infty} \sqrt{\frac{n}{2 \log \log n}\left(\widehat{\beta}_{1}^{(n)}-\beta_{1}\right)} \\
=\sqrt{1-\beta_{1}^{2}}, \quad \text { a.s. }
\end{gathered}
$$

\section{Preliminary Lemmas}

In order to prove Theorem 1, we need the following lemmas.

Lemma 2. Let $\left\{\xi_{n}\right\}_{n \geq 1}$ be a sequence of i.i.d. random variables with $\mathbf{E} \xi_{1}=0$ and $\mathbf{E} \xi_{1}^{2}=\sigma^{2}$. Assume that there a positive constant $\delta>0$ such that $\mathrm{E} e^{\delta\left|\xi_{1}\right|}<\infty$. Then, for any $x>0$,

$$
\begin{aligned}
\lim _{n \rightarrow \infty} & \frac{1}{2 \log \log n} \log \mathbf{P}\left(\left|\frac{\sum_{i=1}^{n} \xi_{i}}{\sqrt{2 n \log \log n}}\right| \geq x\right) \\
& =-\frac{x^{2}}{2 \sigma^{2}} .
\end{aligned}
$$

Proof. Let $\lambda(n) \rightarrow \infty$ and $\lambda(n) / \sqrt{N_{n}} \rightarrow 0$ as $n \rightarrow \infty$. By virtue of the moderate deviation principle for $\left\{\xi_{n}\right\}_{n \geq 1}$ (see pp 109, Dembo and Zeitouni [9]), we have

$$
\limsup _{n \rightarrow \infty} \frac{1}{\lambda^{2}(n)} \log \mathbf{P}\left(\frac{\sum_{i=1}^{n} \xi_{i}}{\sqrt{n} \lambda(n)} \in A\right) \leq-\inf _{x \in A} I(x),
$$

for any closed set $A \subseteq \mathbb{R}$, and

$$
\liminf _{n \rightarrow \infty} \frac{1}{\lambda^{2}(n)} \log \mathbf{P}\left(\frac{\sum_{i=1}^{n} \xi_{i}}{\sqrt{n} \lambda(n)} \in B\right) \geq-\inf _{x \in B} I(x),
$$

for any open set $B \subseteq \mathbb{R}$, where $I(x)=(1 / 2) x^{2} \sigma^{-2}$. Let $A=$ $[x,+\infty)$ and $B=(x,+\infty)$. Set $\lambda(n)=\sqrt{2 \log \log n}$; we easily get the claim by (9) and (10).

The following lemma is about Lévy's inequality. For completeness, we still give its proof.

Lemma 3. Let $\left\{\xi_{n}\right\}_{n \geq 1}$ be a sequence of independent random variables taking their values in $\mathbf{R}$. Let $S_{n}=\sum_{i=1}^{n} \xi_{i}$. Then for any $x>0$,

$$
\mathbf{P}\left(\max _{1 \leq j \leq n}\left(S_{j}+\mathbf{m}\left(S_{n}-S_{j}\right)\right) \geq x\right) \leq 2 \mathbf{P}\left(S_{n} \geq x\right),
$$

where $\mathbf{m}(\xi)$ denotes the median of r.v. X.

Proof. Let $S_{0}=0, M_{k}^{*}=\max _{1 \leq j \leq k}\left(S_{j}+\mathbf{m}\left(S_{n}-\right.\right.$ $\left.\left.S_{j}\right)\right), A_{k}=\left\{M_{k-1}^{*}<x, S_{k}+\mathbf{m}\left(S_{n}-S_{k}\right) \geq x\right\}$, 
and $B_{k}=\left\{S_{n}-S_{k}-\mathbf{m}\left(S_{n}-S_{k}\right) \geq 0\right\}$. From the definition of median, it can be seen that $\mathbf{P}\left(B_{k}\right) \geq 1 / 2$ for any $1 \leq k \leq n$. Further,

$$
\begin{aligned}
\mathbf{P}\left(S_{n} \geq x\right) & \geq \mathbf{P}\left(\bigcup_{k=1}^{n} A_{k} B_{k}\right)=\sum_{k=1}^{n} \mathbf{P}\left(A_{k} B_{k}\right) \\
& =\sum_{k=1}^{n} \mathbf{P}\left(A_{k}\right) \mathbf{P}\left(B_{k}\right) \geq \frac{1}{2} \sum_{k=1}^{n} \mathbf{P}\left(A_{n}\right) \\
& =\frac{1}{2} \mathbf{P}\left(M_{n}^{*} \geq x\right) .
\end{aligned}
$$

Remark 4. A real number $\mathbf{m}(\xi)$ is usually called the median of r.v. $\xi$, if $\mathbf{P}(\xi \geq \mathbf{m}(\xi)) \geq 1 / 2$ and $\mathbf{P}(\xi \leq \mathbf{m}(\xi)) \geq 1 / 2$.

Lemma 5. Let $\left\{\xi_{n}\right\}_{n \geq 1}$ be a sequence of independent random variables with

$$
\begin{aligned}
& \mathbf{E} \xi_{n}=0, \\
& \mathbf{E} \xi_{n}^{2}<\infty .
\end{aligned}
$$

Let $S_{n}=\sum_{i=1}^{n} \xi_{i}$ and $s_{n}^{2}=\mathbf{E} S_{n}^{2}$. Assume the following conditions hold:

$$
\begin{array}{r}
\lim _{n \rightarrow \infty} s_{n}=\infty, \\
\limsup _{n \rightarrow \infty} \frac{s_{n+1}}{s_{n}}<\infty,
\end{array}
$$

and there exists a constant $0<\alpha \leq 1$ satisfying

$$
\sum_{n=1}^{\infty} a_{n}^{-2-\alpha} \mathbf{E}\left|\xi_{n}\right|^{2+\alpha}<\infty
$$

Then $\left\{\xi_{n}\right\}$ satisfies the law of iterated logarithm; that is,

$$
\limsup _{n \rightarrow \infty} \frac{S_{n}}{\sqrt{2 s_{n}^{2} \log \log s_{n}^{2}}}=1, \quad \text { a.s. }
$$

Proof. See Theorem 1.2 of Wittmann [10].

Lemma 6. Let $C_{t, p}=\sum_{i=1}^{p+1} \beta_{1}^{i-1} \epsilon_{t} \epsilon_{t-i}, 1 \leq p \leq t-2$. Then

$$
\begin{aligned}
& \limsup _{p \rightarrow \infty} \limsup _{n \rightarrow \infty} \frac{\sum_{t=1}^{n} C_{t, p}}{\sqrt{2 n \log \log n}}=\sigma^{2} \sqrt{\frac{1}{1-\beta_{1}^{2}}}, \quad \text { a.s., } \\
& \liminf _{p \rightarrow \infty} \liminf _{n \rightarrow \infty} \frac{\sum_{t=1}^{n} C_{t, p}}{\sqrt{2 n \log \log n}}=-\sigma^{2} \sqrt{\frac{1}{1-\beta_{1}^{2}}}, \quad \text { a.s. }
\end{aligned}
$$

Proof. Firstly, note that $\mathbf{E} \epsilon_{t}=0$ and $\mathbf{E}\left[\epsilon_{t} \epsilon_{s}\right]=0$ for any $t \neq s$. So $\mathrm{EC}_{t, p}=0$. Furthermore, for $t \neq s$,

$$
\begin{aligned}
\mathbf{E} & {\left[C_{t, p} C_{s, p}\right] } \\
& =\mathbf{E}\left[\left(\sum_{i=1}^{p+1} \beta_{1}^{i-1} \epsilon_{t} \epsilon_{t-i}\right)\left(\sum_{j=1}^{p+1} \beta_{1}^{j-1} \epsilon_{t} \epsilon_{t-j}\right)\right] \\
& =\sum_{i=1}^{p+1} \sum_{j=1}^{p+1} \beta_{1}^{i+j-2} \mathbf{E}\left[\epsilon_{t} \epsilon_{s} \epsilon_{t-i} \epsilon_{t-j}\right]=0, \\
\mathbf{E} & {\left[C_{t, p}^{2}\right] } \\
& =\mathbf{E}\left[\left(\sum_{i=1}^{p+1} \beta_{1}^{i-1} \epsilon_{t} \epsilon_{t-i}\right)^{2}\right] \\
& =\sum_{i=1}^{p+1} \beta_{1}^{2 i-2} \mathbf{E}\left[\epsilon_{t}^{2} \epsilon_{t-i}^{2}\right]+\sum_{\substack{i, j \in\{1, \ldots, p+1\} \\
i \neq j}} \beta_{1}^{i+j-2} \mathbf{E}\left[\epsilon_{t}^{2} \epsilon_{t-i} \epsilon_{t-j}\right] \\
& =\frac{1-\beta_{1}^{2(p+1)}}{1-\beta_{1}^{2}} \sigma^{4} .
\end{aligned}
$$

Then it is not difficult to see that $\left\{C_{t, p}\right\}_{t \geq 1}$ is a strictly stationary $(p+1)$-dependence sequence of random variables. By (20) and Theorem 1 of Chen [11], one can prove the following equation:

$$
\begin{aligned}
& \limsup _{p \rightarrow \infty} \limsup _{n \rightarrow \infty} \frac{\sum_{t=1}^{n} C_{t, p}}{\sqrt{2 n \log \log n}} \\
& \quad=\limsup _{p \rightarrow \infty}\left\{\mathbf{E} C_{p+2, p}^{2}+2 \sum_{k=p+3}^{2 p+3} \mathbf{E}\left[C_{p+2, p} C_{k, p}\right]\right\}^{1 / 2} \\
& =\sigma^{2} \sqrt{\frac{1}{1-\beta_{1}^{2}}}, \quad \text { a.s. }
\end{aligned}
$$

For the proof of (18), the method is similar.

Lemma 7. Let

$$
\begin{aligned}
A_{n}^{*} & =\sum_{i=0}^{n-2} \sum_{t=i+2}^{n} \beta_{1}^{i} \epsilon_{t} \epsilon_{t-i-1}, \\
A_{n, p} & =\sum_{i=0}^{p} \sum_{t=i+2}^{n} \beta_{1}^{i} \epsilon_{t} \epsilon_{t-i-1} .
\end{aligned}
$$

Then,

$$
\limsup _{p \rightarrow \infty} \limsup _{n \rightarrow \infty} \sqrt{\frac{1}{2 n \log \log n}}\left|A_{n}^{*}-A_{n, p}\right|=0, \quad \text { a.s. }
$$

Proof. In order to prove (23), we only need to show that, for any $\delta>0$ and enough large $p>0$,

$$
\mathbf{P}\left(\left|A_{n}^{*}-A_{n, p}\right| \geq \delta \sqrt{2 n \log \log n} \text {, i.o. }\right)=0,
$$


where i.o. denotes infinitely often occurring. Note that, for any $\tau>0$, there exists an increasing integer sequence $\left\{n_{k}\right\}$ satisfying

$$
n_{k}-1 \leq(1+\tau)^{k}<n_{k}, \quad k \in \mathbb{N} .
$$

So $n_{k}-n_{k-1} \sim\left(n_{k} \tau /(1+\tau)\right)$, where $a_{n} \sim b_{n}$ denotes $a_{n} / b_{n} \rightarrow 1$. Furthermore, for any $\delta>0$, we have the following inequality:

$$
\begin{aligned}
& \mathbf{P}\left(\left|A_{n}^{*}-A_{n, p}\right| \geq \delta \sqrt{2 n \log \log n}, \text { i.o. }\right) \\
& \leq \mathbf{P}\left(\max _{n_{k-1} \leq n<n_{k}}\left|A_{n}^{*}-A_{n, p}\right| \geq \delta \sqrt{2 n_{k-1} \log \log n_{k-1}}, \text { i.o. }\right) \\
& \leq \mathbf{P}\left(\max _{n \leq n_{k}}\left|A_{n}^{*}-A_{n, p}\right| \geq \delta \sqrt{2 n_{k-1} \log \log n_{k-1}}, \text { i.o. }\right) .
\end{aligned}
$$

Also note that, for $n_{k}$ enough large,

$$
\frac{\sqrt{2 n_{k} \log \log n_{k}}}{\sqrt{2 n_{k-1} \log \log n_{k-1}}}<\sqrt{1+2 \tau}
$$

So

$$
\begin{aligned}
& \mathbf{P}\left(\left|A_{n}^{*}-A_{n, p}\right| \geq \delta \sqrt{2 n \log \log n} \text {, i.o. }\right) \\
& \quad \leq \mathbf{P}\left(\max _{n \leq n_{k}}\left|A_{n}^{*}-A_{n, p}\right| \geq \frac{\delta}{\sqrt{1+2 \tau}} \sqrt{2 n_{k} \log \log n_{k}} \text {, i.o. }\right) .
\end{aligned}
$$

Set $r>0$ and let $\tau>0$ satisfy $\delta / \sqrt{1+2 \tau}>r$. Thus

$$
\begin{aligned}
& \mathbf{P}\left(\left|A_{n}^{*}-A_{n, p}\right| \geq \delta \sqrt{2 n \log \log n} \text {, i.o. }\right) \\
& \quad \leq \mathbf{P}\left(\max _{n \leq n_{k}}\left|A_{n}^{*}-A_{n, p}\right| \geq r \sqrt{2 n_{k} \log \log n_{k}} \text {, i.o. }\right) .
\end{aligned}
$$

Since $\left|\beta_{1}\right|<1$, one has

$$
K_{1}:=\sum_{i=p+1}^{+\infty}\left|\beta_{1}\right|^{i-p}(i+1)<\infty .
$$

Consequently,

$$
\begin{gathered}
\mathbf{P}\left(\max _{n \leq n_{k}}\left|A_{n}^{*}-A_{n, p}\right|>r \sqrt{2 n_{k} \log \log n_{k}}\right) \\
\leq \mathbf{P}\left(\sum_{i=p+1}^{n-2}\left|\beta_{1}\right|^{i-p} \max _{n \leq n_{k}}\left|\sum_{t=i+2}^{n} \epsilon_{t} \epsilon_{t-i-1}\right|\right. \\
\left.>\sum_{i=p+1}^{n-2}\left|\beta_{1}\right|^{i-p}(i+1) \frac{\left(1-\left|\beta_{1}\right|\right) r \sqrt{2 n_{k} \log \log n_{k}}}{K_{1}\left|\beta_{1}\right|^{p+1}}\right) \\
\leq \sum_{i=p+1}^{n-2} \mathbf{P}\left(\max _{n \leq n_{k}}\left|\sum_{t=i+2}^{n} \epsilon_{t} \epsilon_{t-i-1}\right|\right. \\
\left.>\frac{(i+1)\left(1-\left|\beta_{1}\right|\right) r \sqrt{2 n_{k} \log \log n_{k}}}{K_{1}\left|\beta_{1}\right|^{p+1}}\right) .
\end{gathered}
$$

For any positive natural numbers $i, p$, and $s$, define

$$
\begin{aligned}
A_{i+1} & (n) \\
\quad & \{2 q(i+1)+j: q \in \mathbb{N}, 1 \leq j \leq i+1\} \\
& \cap\{1, \ldots, n\}, \\
B_{i+1} & (n) \\
\quad & \{(2 q+1)(i+1)+j: q \in \mathbb{N}, 1 \leq j \leq i+1\} \\
& \cap\{1, \ldots, n\} .
\end{aligned}
$$

It is easy to see that $\left\{\epsilon_{t} \epsilon_{t-i-1}\right\}_{t \in A_{i+1}(n)}$ and $\left\{\epsilon_{t} \epsilon_{t-i-1}\right\}_{t \in B_{i+1}(n)}$ are two sets of i.i.d. random variables. Let $\left\{\widetilde{\epsilon}_{t} \widetilde{\epsilon}_{t-i-1}\right\}$ be an i.i.d. sequence of random variables with the same law as $\epsilon_{t} \epsilon_{t-i-1}$. We have

$$
\begin{gathered}
\mathbf{P}\left(\max _{n \leq n_{k}}\left|A_{n}^{*}-A_{n, p}\right|>r \sqrt{2 n_{k} \log \log n_{k}}\right) \\
\leq \sum_{i=p+1}^{n-2} \mathbf{P}\left(\max _{n \leq n_{k}}\left|\sum_{t \in A_{i+1}(n)} \widetilde{\epsilon}_{\epsilon} \widetilde{\epsilon}_{t-i-1}\right|\right. \\
\left.>\frac{(i+1)\left(1-\left|\beta_{1}\right|\right) r \sqrt{2 n_{k} \log \log n_{k}}}{2 K_{1}\left|\beta_{1}\right|^{p+1}}\right) \\
+\sum_{i=p+1}^{n-2} \mathbf{P}\left(\max _{n \leq n_{k}}\left|\sum_{t \in B_{i+1}(n)} \widetilde{\epsilon}_{t} \widetilde{\epsilon}_{t-i-1}\right|\right. \\
\left.>2 \frac{(i+1)\left(1-\left|\beta_{1}\right|\right) r \sqrt{2 n_{k} \log \log n_{k}}}{2 K_{1}\left|\beta_{1}\right|^{p+1}}\right) \\
\leq \sum_{i=p+1}^{n-2} \mathbf{P}\left(\max _{n \leq n_{k}}\left|\sum_{t=i+2}^{n} \widetilde{\epsilon}_{t} \widetilde{\epsilon}_{t-i-1}\right|\right. \\
\left.>\frac{(i+1)\left(1-\left|\beta_{1}\right|\right) r \sqrt{2 n_{k} \log \log n_{k}}}{2 K_{1}\left|\beta_{1}\right|^{p+1}}\right) .
\end{gathered}
$$

Note that for any random variable $X$ with mean zero, $|\mathbf{m}(X)| \leq \sqrt{2 \operatorname{Var} X}$. In fact,

$$
\mathbf{P}(|X| \geq \sqrt{(2+\epsilon) \operatorname{Var} X}) \leq(2+\epsilon)^{-1}<\frac{1}{2} .
$$

By Lemma 3,

$$
\begin{aligned}
\mathbf{P}\left(\max _{n \leq n_{k}}\left|\sum_{t=i+2}^{n} \widetilde{\epsilon}_{t} \widetilde{\epsilon}_{t-i-1}\right|\right. \\
\left.>\frac{(i+1)\left(1-\left|\beta_{1}\right|\right) r \sqrt{2 n_{k} \log \log n_{k}}}{2 K_{1}\left|\beta_{1}\right|^{p+1}}\right)
\end{aligned}
$$




$$
\begin{array}{r}
\leq 2 \mathbf{P}\left(\left|\sum_{t=i+2}^{n_{k}} \widetilde{\epsilon}_{t} \widetilde{\epsilon}_{t-i-1}\right|\right. \\
\left.>\frac{(i+1)\left(1-\left|\beta_{1}\right|\right) r \sqrt{2 n_{k} \log \log n_{k}}}{2 K_{1}\left|\beta_{1}\right|^{p+1}}-\sqrt{2 n_{k} \sigma^{4}}\right) \\
\leq 2 \mathbf{P}\left(\left|\sum_{t=i+2}^{n_{k}} \widetilde{\epsilon}_{t} \widetilde{\epsilon}_{t-i-1}\right|\right. \\
\left.>\frac{(i+1)\left(1-\left|\beta_{1}\right|\right) r_{1} \sqrt{2 n_{k} \log \log n_{k}}}{2 K_{1}\left|\beta_{1}\right|^{p+1}}\right),
\end{array}
$$

where the second inequality holds for any fixed constant $r_{1}>$ 0 and enough large $n_{k}$. By Lemma 2, we further have

$$
\begin{aligned}
& \mathbf{P}\left(\max _{n \leq n_{k}}\left|A_{n}^{*}-A_{n, p}\right|>r \sqrt{2 n_{k} \log \log n_{k}}\right) \\
& \leq 4 \sum_{i=1}^{\infty} \exp \left\{-\frac{(i+1)\left(1-\left|\beta_{1}\right|\right)^{2} r_{1}^{2}}{\left.4 K_{1}^{2}\left|\beta_{1}\right|^{2 p+2} \log \log n_{k}\right\}}\right. \\
& \leq \frac{4 \exp \left\{-\left(2\left(1-\left|\beta_{1}\right|\right)^{2} r_{1}^{2} / 4 K_{1}^{2}\left|\beta_{1}\right|^{2 p+2}\right) \log \log n_{k}\right\}}{1-\exp \left\{-\left(\left(1-\left|\beta_{1}\right|\right)^{2} r_{1}^{2} / 4 K_{1}^{2}\left|\beta_{1}\right|^{2 p+2}\right) \log \log n_{k}\right\}} \\
& \sim 4 \exp \left\{-\frac{\left(1-\left|\beta_{1}\right|\right)^{2} r_{1}^{2}}{2 K_{1}^{2}\left|\beta_{1}\right|^{2 p+2}} \log \log n_{k}\right\} .
\end{aligned}
$$

From $(25), n_{k} \sim(1+\tau)^{k}$ as $k \rightarrow \infty$. So

$$
\begin{gathered}
\mathbf{P}\left(\max _{n \leq n_{k}}\left|A_{n}^{*}-A_{n, p}\right|>r \sqrt{2 n_{k} \log \log n_{k}}\right) \\
\quad \leq 4\left(\frac{1}{k \log (1+\tau)}\right)^{\left(1-\left|\beta_{1}\right|^{2}\right) r_{1}^{2} / 2 K_{1}^{2}\left|\beta_{1}\right|^{2 p+2}} .
\end{gathered}
$$

By virtue of $\left|\beta_{1}\right|<1$ and $p \rightarrow+\infty$, we easily see that $(1-$ $\left.\left|\beta_{1}\right|^{2}\right) r_{1}^{2} / 2 K_{1}^{2}\left|\beta_{1}\right|^{2 p+2}>1$. Thus

$$
\sum_{k=1}^{\infty} \mathbf{P}\left(\max _{n \leq n_{k}}\left|A_{n}^{*}-A_{n, p}\right|>r \sqrt{2 n_{k} \log \log n_{k}}\right)<+\infty .
$$

Applying Borel-Cantelli Lemma, we get (24). This completes the proof.

Lemma 8. Let $B_{n}^{(1)}=\sum_{t=1}^{n} X_{t-1}$ and $B_{n}^{(2)}=\sum_{t=1}^{n} X_{t-1}^{2}$. Then

$$
\begin{aligned}
& \lim _{n \rightarrow \infty} \frac{B_{n}^{(1)}}{n}=\frac{\beta_{0}}{1-\beta_{1}}, \quad \text { a.s., } \\
& \lim _{n \rightarrow \infty} \frac{B_{n}^{(2)}}{n}=\frac{\beta_{0}^{2}}{\left(1-\beta_{1}\right)^{2}}+\frac{\sigma^{2}}{1-\beta_{1}^{2}}, \quad \text { a.s. }
\end{aligned}
$$

Proof. From (1), one can easily calculate

$$
X_{t-1}=\frac{\beta_{0}\left(1-\beta_{1}^{t-1}\right)}{1-\beta_{1}}+\beta_{1}^{t-1} X_{0}+\sum_{i=1}^{t-1} \beta_{1}^{t-1-i} \epsilon_{i}, \quad t \geq 2 .
$$

Consequently,

$$
\begin{aligned}
& B_{n}^{(1)} \\
& =X_{0} \sum_{t=1}^{n} \beta_{1}^{t-1}+\beta_{0} \sum_{t=2}^{n} \frac{1-\beta_{1}^{t-1}}{1-\beta_{1}} \\
& +\left[\epsilon_{1}\left(1+\beta_{1}+\cdots+\beta_{1}^{n-2}\right)\right. \\
& \left.\quad+\epsilon_{2}\left(1+\beta_{1}+\cdots+\beta_{1}^{n-3}\right)+\cdots+\epsilon_{n-1}\right] .
\end{aligned}
$$

Now for any $n \geq k+1$,

$$
\mathbf{E}\left[\epsilon_{k}^{2}\left(1+\beta_{1}+\cdots+\beta_{1}^{n-k-1}\right)^{2}\right] \leq \frac{\sigma^{2}}{\left(1-\beta_{1}\right)^{2}},
$$

so the bracket of (41) divided by $n$ converges a.e. to 0 , thanks to Theorem 5.4.1 of Chung [12]. Furthermore,

$$
\begin{aligned}
\lim _{n \rightarrow \infty} \frac{X_{0}\left(1+\beta_{1}+\cdots+\beta_{1}^{n-1}\right)}{n} & =0, \quad \text { a.s., } \\
\lim _{n \rightarrow \infty} \frac{\beta_{0}}{n} \sum_{k=2}^{n} \frac{1-\beta_{1}^{k-1}}{1-\beta_{1}} & =\frac{\beta_{0}}{1-\beta_{1}} .
\end{aligned}
$$

The former equality of (39) is obtained.

As to the later one, $B_{n}^{(2)}$ can be written as

$$
\begin{aligned}
& \beta_{0}^{2} \sum_{t=2}^{n}\left(\frac{1-\beta_{1}^{t-1}}{1-\beta_{1}}\right)^{2}+X_{0}^{2} \sum_{t=1}^{n} \beta_{1}^{2(t-1)} \\
& \quad+2 X_{0} \beta_{0} \sum_{t=2}^{n} \beta_{1}^{t-1} \frac{1-\beta_{1}^{t-1}}{1-\beta_{1}}+2 X_{0} \sum_{t=2}^{n} \beta_{1}^{t-1}\left(\sum_{i=1}^{t-1} \beta_{1}^{t-1-i} \epsilon_{i}\right) \\
& +\sum_{t=2}^{n}\left(\sum_{i=1}^{t-1} \beta_{1}^{t-1-i} \epsilon_{i}\right)^{2} .
\end{aligned}
$$

Note that $\left|\beta_{1}\right|<1$, one can see that

$$
\begin{aligned}
\lim _{n \rightarrow \infty} \frac{1}{n} \beta_{0}^{2} \sum_{t=2}^{n}\left(\frac{1-\beta_{1}^{t-1}}{1-\beta_{1}}\right)^{2} \\
=\frac{\beta_{0}^{2}}{\left(1-\beta_{1}\right)^{2}}
\end{aligned}
$$

$$
\begin{aligned}
& \lim _{n \rightarrow \infty} \frac{1}{n}\left[X_{0}^{2} \sum_{t=1}^{n} \beta_{1}^{2(t-1)}+2 X_{0} \beta_{0} \sum_{t=2}^{n} \beta_{1}^{t-1} \frac{1-\beta_{1}^{t-1}}{1-\beta_{1}}\right] \\
& \quad=0 .
\end{aligned}
$$

The next to last term of (44) equals $2 X_{0} \sum_{i=1}^{n-1} \epsilon_{i} \sum_{j=0}^{n-1-i} \beta_{1}^{i+2 j}$, which yields the fact that the mean is zero and variance is bounded by constant times $n$. Thus

$$
\lim _{n \rightarrow \infty} \frac{2 X_{0}}{n} \sum_{t=2}^{n} \beta_{1}^{t-1} \sum_{i=1}^{t-1} \beta_{1}^{t-1-i} \epsilon_{i}=0
$$


The last step is to prove

$$
\lim _{n \rightarrow \infty} \frac{1}{n} \sum_{t=2}^{n}\left(\sum_{i=1}^{t-1} \beta_{1}^{t-1-i} \epsilon_{i}\right)^{2}=\frac{\sigma^{2}}{1-\beta_{1}^{2}}, \quad \text { a.s. }
$$

By a simple calculation, one has

$$
\begin{aligned}
\sum_{t=2}^{n}\left(\sum_{i=1}^{t-1} \beta_{1}^{t-1-i} \epsilon_{i}\right)^{2} & \\
= & \sum_{t=1}^{n-1} \epsilon_{t}^{2} \sum_{i=0}^{n-1-t} \beta_{1}^{2 i} \\
& +2 \sum_{j=1}^{n-2} \epsilon_{j} \sum_{i=j+1}^{n-1} \epsilon_{i} \frac{\beta_{1}^{i-j}\left(1-\beta_{1}^{2(n-i)}\right)}{1-\beta_{1}^{2}} .
\end{aligned}
$$

Note that $\left\{\epsilon_{t}\right\}_{t \geq 1}$ is a sequence of i.i.d. random variables and

$$
\sum_{i=0}^{n-1-t} \beta_{1}^{2 i}=\frac{1-\beta_{1}^{2(n-t)}}{1-\beta_{1}^{2}}
$$

By virtue of the strong law of large numbers on independent random variables (see Chung [12]), one has

$$
\begin{aligned}
\lim _{n \rightarrow \infty} \frac{1}{n} \sum_{t=1}^{n-1} \epsilon_{t}^{2} \sum_{i=0}^{n-1-t} \beta_{1}^{2 i} & =\lim _{n \rightarrow \infty} \frac{\sigma^{2}}{n} \sum_{t=1}^{n-1} \sum_{i=0}^{n-1-t} \beta_{1}^{2 i} \\
& =\frac{\sigma^{2}}{1-\beta_{1}^{2}}, \quad \text { a.s. }
\end{aligned}
$$

The mean of the third term in (48) is zero and the variance is less than

$$
4 \sigma^{4} \sum_{j=1}^{n-2} \sum_{i=j+1}^{n-1} \frac{\beta_{1}^{2 i-2 j}}{\left(1-\beta_{1}^{2}\right)^{2}} \leq \frac{4 n \sigma^{4} \beta_{1}^{2}}{\left(1-\beta_{1}^{2}\right)^{3}}
$$

which yields

$$
\lim _{n \rightarrow \infty} \frac{2}{n} \sum_{j=1}^{n-2} \epsilon_{j} \sum_{i=j+1}^{n-1} \epsilon_{i} \frac{\beta_{1}^{i-j}\left(1-\beta_{1}^{2(n-i)}\right)}{1-\beta_{1}^{2}}=0, \quad \text { a.s. }
$$

So the proof of the lemma is completed.

\section{Proof of Theorem 1}

The proof is divided into four steps as follows.

Step 1. Let

$$
A_{n}=\sum_{t=1}^{n} \epsilon_{t} X_{t-1}
$$

Then $A_{n}$ can be calculated by

$$
\begin{aligned}
A_{n}= & \epsilon_{1} X_{0}+\sum_{t=2}^{n} \epsilon_{t} \beta_{0}+\sum_{t=2}^{n} \epsilon_{t} \epsilon_{t-1}+\sum_{t=2}^{n} \beta_{1} \epsilon_{t} X_{t-2} \\
= & \cdots \\
= & \sum_{i=0}^{n-2} \sum_{t=i+2}^{n} \beta_{1}^{i} \epsilon_{t} \epsilon_{t-i-1} \\
& +\beta_{0} \sum_{i=0}^{n-2} \sum_{t=i+2}^{n} \beta_{1}^{i} \epsilon_{t}+X_{0} \sum_{t=1}^{n} \beta_{1}^{t-1} \epsilon_{t} \\
:= & A_{n}^{*}+\beta_{0} B_{n}^{*}+X_{0} \sum_{t=1}^{n} \beta_{1}^{t-1} \epsilon_{t} .
\end{aligned}
$$

It is easily seen that

$$
\limsup _{n \rightarrow \infty} \frac{\left|X_{0} \sum_{t=1}^{n} \beta_{1}^{t-1} \epsilon_{t}\right|}{\sqrt{2 n \log \log n}}=0, \quad \text { a.s. }
$$

Now we come to prove

$$
\limsup _{n \rightarrow \infty} \frac{A_{n}^{*}}{\sqrt{2 n \log \log n}}=\frac{\sigma^{2}}{\sqrt{1-\beta_{1}^{2}}}, \quad \text { a.s. }
$$

Indeed, set a nature number $p$ and define

$$
\begin{array}{r}
A_{n, p} \\
= \begin{cases}A_{n, p}=A_{n}^{*}, & \text { if } p>n-2, \\
\sum_{i=0}^{p} \sum_{t=i+2}^{n} \beta_{1}^{i} \epsilon_{t} \epsilon_{t-i-1}, & \text { if } p \leq n-2 .\end{cases}
\end{array}
$$

Note that $A_{n}^{*}$ is a linear combination of terms $\epsilon_{t}(j) \epsilon_{t-s}(t(j))$ and $\mathbf{E}\left[\epsilon_{t}(j) \epsilon_{t-s}(t(j))\right]=0$ for any $s<t$; we easily obtain $\mathbf{E}\left[A_{n}^{*}-A_{n, p}\right]=0$. Furthermore, from Lemma 7,

$$
\limsup _{p \rightarrow \infty} \limsup _{n \rightarrow \infty} \sqrt{\frac{1}{2 n \log \log n}}\left|A_{n}^{*}-A_{n, p}\right|=0, \quad \text { a.s. }
$$

Next, we let

$$
C_{t, p}^{*}=\sum_{t=p+2}^{n} C_{t, p}=\sum_{t=p+2}^{n} \sum_{i=0}^{p} \beta_{1}^{i} \epsilon_{t} \epsilon_{t-i-1} .
$$

Then the limit distribution of $C_{t, p}^{*} / \sqrt{2 n \log \log n}$ is the same as that of $A_{n, p} / \sqrt{2 n \log \log n}$. Further,

$$
\limsup _{p \rightarrow \infty} \limsup _{n \rightarrow \infty} \frac{1}{\sqrt{2 n \log \log n}}\left|A_{n, p}-C_{t, p}^{*}\right|=0, \quad \text { a.s. }
$$

From (58) and (60), combining Lemma 6, we get (56).

Step 2. We claim that

$$
\limsup _{n \rightarrow \infty} \frac{\left|B_{n}^{*}-\left(\sum_{t=1}^{n} X_{t-1}\right)\left(\sum_{t=1}^{n} \epsilon_{t}\right)\right|}{n \sqrt{2 n \log \log n}}=0, \quad \text { a.s. }
$$


Note that

$$
B_{n}^{*}=\sum_{i=0}^{n-2} \sum_{t=i+2}^{n} \beta_{1}^{i} \epsilon_{t}=\sum_{t=2}^{n} \frac{1-\beta_{1}^{t-1}}{1-\beta_{1}} \epsilon_{t}
$$

and $\lim _{n \rightarrow \infty}\left(\left(\sum_{t=1}^{n} X_{t-1}\right) / n\right)=\left(\beta_{0} /\left(1-\beta_{1}\right)\right)$; we have

$$
\begin{aligned}
& \limsup _{n \rightarrow \infty} \frac{\left|B_{n}^{*}-\left(\sum_{t=1}^{n} X_{t-1}\right)\left(\sum_{t=1}^{n} \epsilon_{t}\right)\right|}{n \sqrt{2 n \log \log n}} \\
& \quad=\limsup _{n \rightarrow \infty} \frac{\left|\sum_{t=2}^{n}\left(\beta_{0} \beta_{1}^{t-1} /\left(1-\beta_{1}\right)\right) \epsilon_{t}\right|}{\sqrt{2 n \log \log n}} .
\end{aligned}
$$

Since $\left\{\epsilon_{t}\right\}_{t \geq 1}$ is a sequence of i.i.d. random variables, one easily checks that the sequence $\left\{\left(\beta_{0} \beta_{1}^{t-1} /\left(1-\beta_{1}\right)\right) \epsilon_{t}\right\}$ satisfies the conditions of Lemma 5. Thus, (61) is obtained.

Step 3. We claim that

$$
\begin{aligned}
& \limsup _{n \rightarrow \infty} \frac{-\left(\sum_{t=1}^{n} X_{t-1}\right)\left(\sum_{t=1}^{n} \epsilon_{t}\right)+n \sum_{t=1}^{n} \epsilon_{t} X_{t-1}}{n \sqrt{2 n \log \log n}} \\
& =\frac{\sigma^{2}}{\sqrt{1-\beta_{1}^{2}}}, \quad \text { a.s., } \\
& \limsup _{n \rightarrow \infty} \frac{\left(\sum_{t=1}^{n} X_{t-1}^{2}\right)\left(\sum_{t=1}^{n} \epsilon_{t}\right)-\left(\sum_{t=1}^{n} X_{t-1}\right)\left(\sum_{t=1}^{n} \epsilon_{t} X_{t-1}\right)}{n \sqrt{2 n \log \log n}} \\
& \quad=\sqrt{\frac{\sigma^{6}}{\left(1-\beta_{1}^{2}\right)^{2}}+\left(\frac{\beta_{0}}{1-\beta_{1}}\right)^{2} \cdot \frac{\sigma^{4}}{1-\beta_{1}^{2}}}, \text { a.s. }
\end{aligned}
$$

By (54), (55), and (61), we easily get (64). By virtue of Lemma $8,(65)$ is equivalent to

$$
\begin{gathered}
\limsup _{n \rightarrow \infty}(( \\
\left.\cdot \sum_{t=1}^{n} \epsilon_{t}-\frac{\beta_{0}}{1-\beta_{1}}\left(\sum_{t=1}^{n} \epsilon_{t} X_{t-1}\right)\right) \\
\left.\cdot(\sqrt{2 n \log \log n})^{-1}\right) \\
=\sqrt{\frac{\sigma^{6}}{\left(1-\beta_{1}^{2}\right)^{2}}+\left(\frac{\beta_{0}}{1-\beta_{1}}\right)^{2} \cdot \frac{\sigma^{4}}{1-\beta_{1}^{2}}}, \text { a.s. }
\end{gathered}
$$

From (63), we can see that

$$
\limsup _{n \rightarrow \infty} \frac{\left|\left(\beta_{0}^{2} /\left(1-\beta_{1}\right)^{2}\right) \sum_{t=1}^{n} \epsilon_{t}-\left(\beta_{0}^{2} /\left(1-\beta_{1}\right)\right) \sum_{i=0}^{n-2} \sum_{t=i+2}^{n} \beta_{1}^{i} \epsilon_{t}\right|}{\sqrt{2 n \log \log n}}
$$$$
=0, \quad \text { a.s }
$$

So from (58) and (60), (65) is also equivalent to

$$
\begin{gathered}
\limsup _{n \rightarrow \infty} \frac{\sum_{t=1}^{n}\left(\left(\sigma^{2} /\left(1-\beta_{1}^{2}\right)\right)-\left(\beta_{0} /\left(1-\beta_{1}\right)\right) \sum_{i=1}^{p+1} \beta_{1}^{i-1} \epsilon_{t-i}\right) \epsilon_{t}}{\sqrt{2 n \log \log n}} \\
\quad=\sqrt{\frac{\sigma^{6}}{\left(1-\beta_{1}^{2}\right)^{2}}+\left(\frac{\beta_{0}}{1-\beta_{1}}\right)^{2} \cdot \frac{\sigma^{4}}{1-\beta_{1}^{2}}}, \quad \text { a.s. }
\end{gathered}
$$

Similarly to the proof of Lemma 6, (68) can be easily derived.

Step 4. Since

$$
\begin{aligned}
& \sqrt{\frac{n}{2 \log \log n}\left(\hat{\beta}_{0}^{(n)}-\beta_{0}\right)} \\
= & \frac{\left(\sum_{t=1}^{n} X_{t-1}^{2}\right)\left(\sum_{t=1}^{n} \epsilon_{t}\right)-\left(\sum_{t=1}^{n} X_{t-1}\right)\left(\sum_{t=1}^{n} \epsilon_{t} X_{t-1}\right)}{n \sqrt{2 n \log \log n}} \\
& \cdot \frac{\left(\frac{\sum_{t=1}^{n} X_{t-1}^{2}}{n}-\left(\frac{\sum_{t=1}^{n} X_{t-1}}{n}\right)^{2}\right)^{-1}}{2 \log \log n}\left(\hat{\beta}_{1}^{(n)}-\beta_{1}\right) \\
= & \frac{-\left(\sum_{t=1}^{n} X_{t-1}\right)\left(\sum_{t=1}^{n} \epsilon_{t}\right)+n \sum_{t=1}^{n} \epsilon_{t} X_{t-1}}{n \sqrt{2 n \log \log n}} \\
& \cdot\left(\frac{\sum_{t=1}^{n} X_{t-1}^{2}}{n}-\left(\frac{\sum_{t=1}^{n} X_{t-1}}{n}\right)^{2}\right)^{-1} .
\end{aligned}
$$

By Lemma 8, together with (64) and (65), we obtain (7). This completes the proof of the theorem.

\section{Conflict of Interests}

The authors declare that there is no conflict of interests regarding the publication of this paper.

\section{Acknowledgments}

This work is supported by the Major Project of National Social Science Fund (12\&2D223), by the Program of Introducing Talents of Discipline to Universities (B07042), and in part by NSF IIP 1160960 and NNS IIP 1332024.

\section{References}

[1] H. B. Mann and A. Wald, "On the statistical treatment of linear stochastic difference equations," Econometrica, vol. 11, pp. 173220, 1943.

[2] T. W. Anderson, "On asymptotic distributions of estimates of parameters of stochastic difference equations," Annals of Mathematical Statistics, vol. 30, pp. 676-687, 1959.

[3] L. Menneteau, "Some laws of the iterated logarithm in Hilbertian autoregressive models," Journal of Multivariate Analysis, vol. 92, no. 2, pp. 405-425, 2005. 
[4] S. Y. Hwang and M. S. Choi, "Modeling and large sample estimation for multi-casting autoregression," Statistics \& Probability Letters, vol. 79, no. 18, pp. 1943-1950, 2009.

[5] S. Y. Hwang and J. S. Baek, "Limiting mixture distributions for AR(1) model indexed by a branching process," Statistics \& Probability Letters, vol. 80, no. 23-24, pp. 2003-2008, 2010.

[6] Y. Miao and S. Shen, "Moderate deviation principle for autoregressive processes," Journal of Multivariate Analysis, vol. 100, no. 9, pp. 1952-1961, 2009.

[7] S. P. Meyn and R. L. Tweedie, Markov Chains and Stochastic Stability, Springer, New York, NY, USA, 2nd edition, 1993.

[8] I. V. Basawa and P. J. Brockwell, "Asymptotic conditional inference for regular nonergodic models with an application to autoregressive processes," The Annals of Statistics, vol. 12, no. 1, pp. 161-171, 1984.

[9] A. Dembo and O. Zeitouni, Large Deviations Techniques and Applications, Springer, New York, NY, USA, 2nd edition, 1998.

[10] R. Wittmann, "A general law of iterated logarithm," Zeitschrift für Wahrscheinlichkeitstheorie und Verwandte Gebiete, vol. 68, no. 4, pp. 521-543, 1985.

[11] X. Chen, "The law of the iterated logarithm for $m$-dependent Banach space valued random variables," Journal of Theoretical Probability, vol. 10, no. 3, pp. 695-732, 1997.

[12] K. L. Chung, A Course in Probability Theory, Springer, New York, NY, USA, 2nd edition, 1974. 


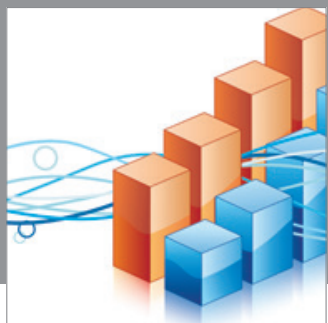

Advances in

Operations Research

mansans

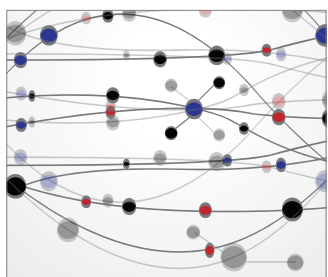

The Scientific World Journal
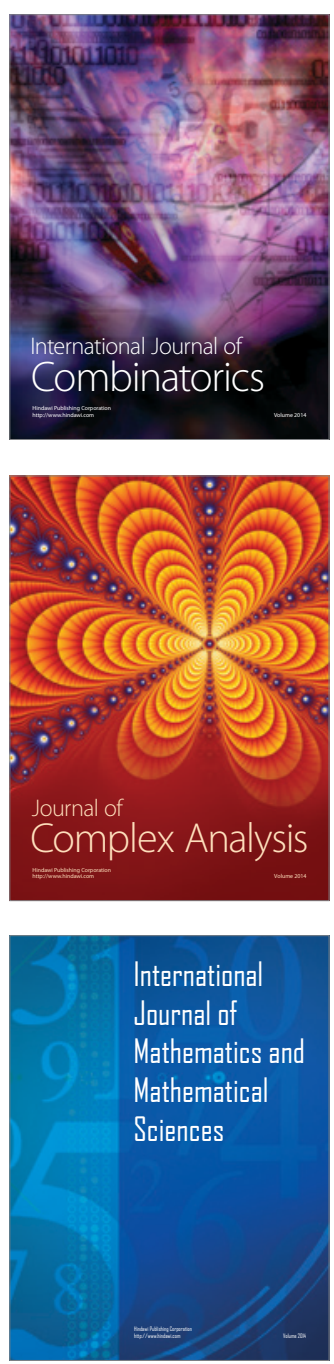
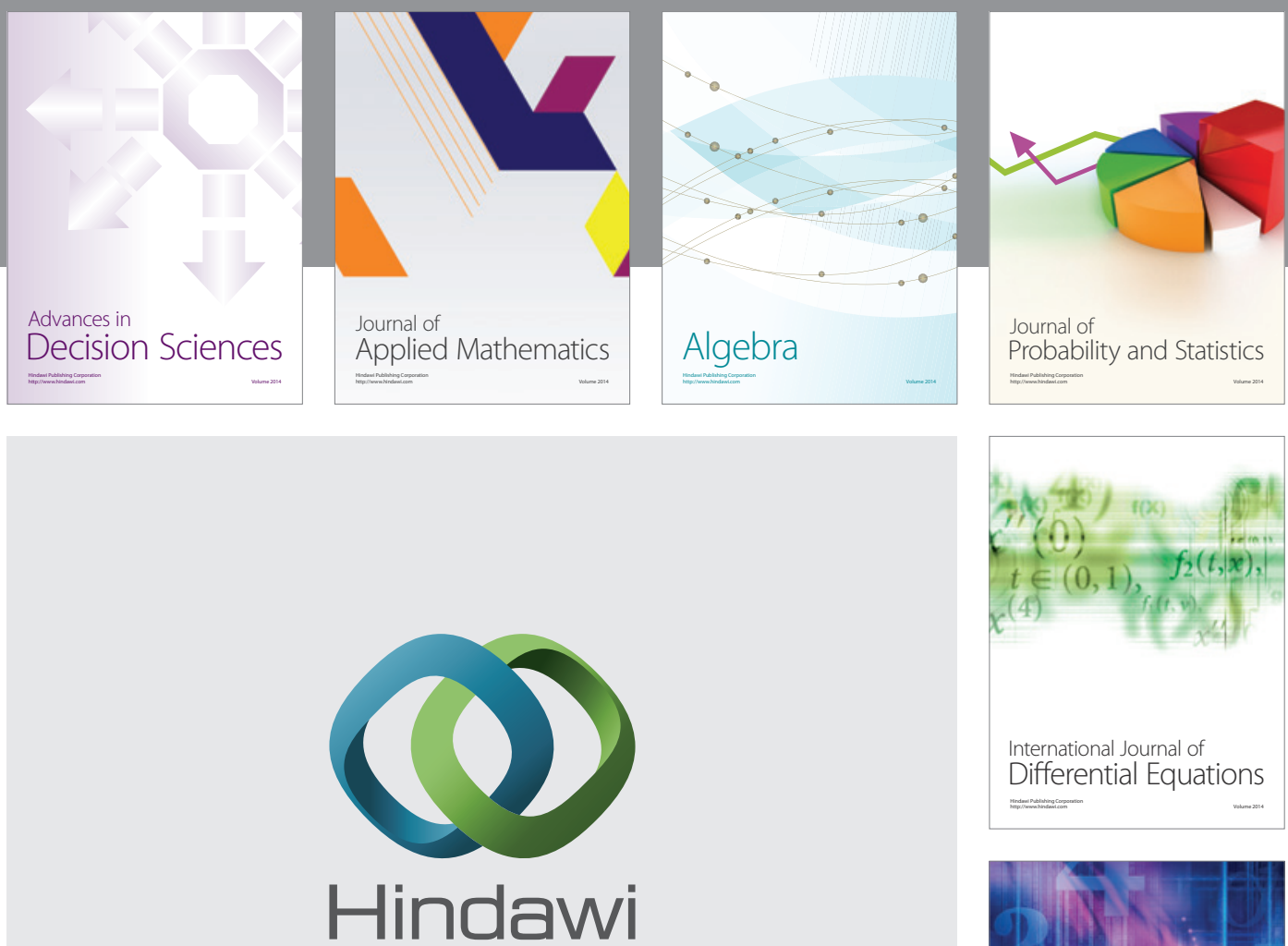

Submit your manuscripts at http://www.hindawi.com
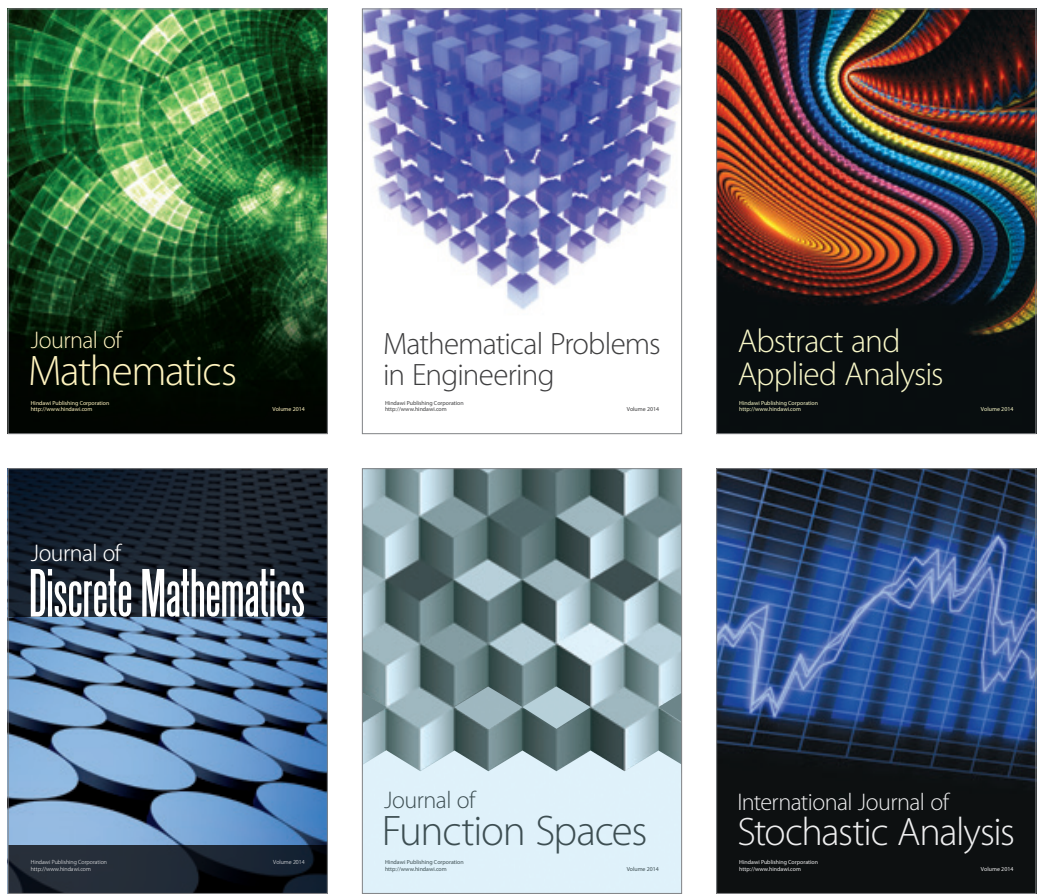

Journal of

Function Spaces

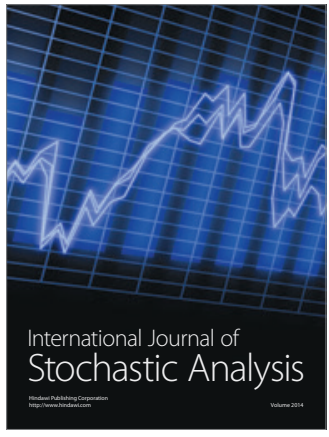

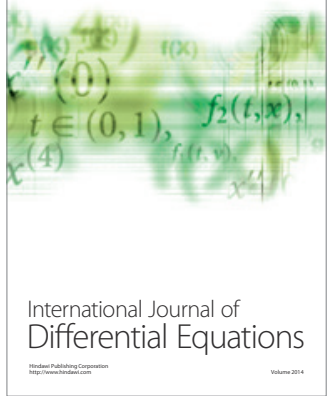
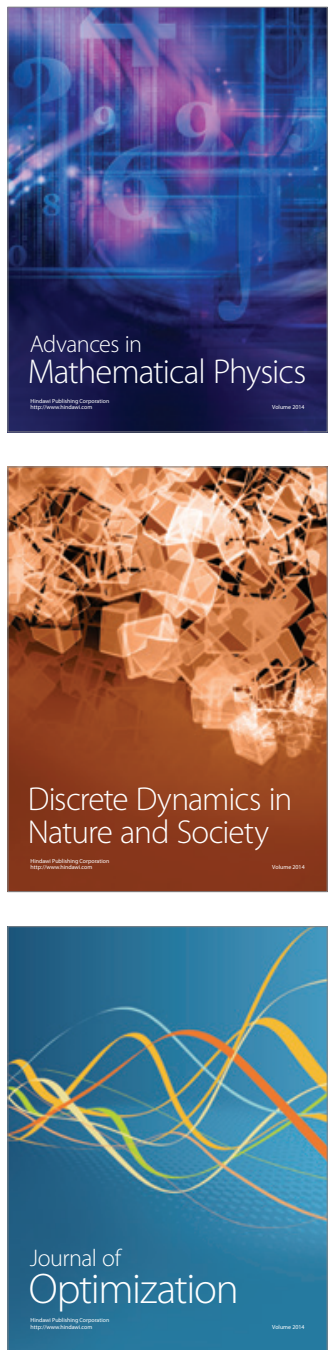